Z. klin. Chem. u. klin. Biochem.

7. Jg., S. 518-520, September 1969

\title{
Über die Hydrolyse von $\beta$-Propiolacton in Seren und Plasmen
}

\author{
Von W. STEPHAN \\ Aus der Wissenschaftlichen Abteilung der Biotest-Serum-Institut GmbH, Frankfurt (Main) \\ (Leiter: Privatdozent Dr. H. Determann)
}

(Eingegangen am 3. Juni 1969)

\begin{abstract}
Die von LoGRIPpo empfohlene Methode zur Herstellung von hepatitissicheren Seren und Plasmen kann ohne Reduzierung der $\beta$-Propiolacton-Konzentration nur in Serum- und Plasmapools mit genügender Esterase-Aktivität angewendet werden, wenn man Proteinmodifizierungen vermeiden will. In solchen Seren und Plasmen verläuft die Umwandlung des $\beta$-Propiolacton zu $\beta$-Hydroxypropionsäure rasch, so daß nach kurzer Zeit kein freies $\beta$-Propiolacton in den behandelten Seren und Plasmen vorliegt. Das Ende dieser Hydrolyse ist leicht ablesbar an der Konstanz des pH-Wertes.

Faktoren, die die Esterase-Aktivität und Hydrolysegeschwindigkeit von $\beta$-Propiolacton herabsetzen und somit Veranlassung geben zu Proteinmodifizierungen, sind u. a. Erhitzen, Adsorption und starke Komplexbildner wie EDTA. EDTA-Plasmen verlieren aus diesem Grund bei Behandlung mit 0,3\% $\beta$-Propiolacton ihre Gerinnungsaktivität.
\end{abstract}

\section{The bydrolysis of $\beta$-propiolactone in serum and plasma}

The method for the removel of hepatitis virus from sera amd plasmas with $\beta$-propiolactone according to LoGRIPpo can only be used without modifying proteins if the treated sera contain sufficient esterase activity normally present in serum pools.

In the latter type of sera the conversion of $\beta$-propiolactone into $\beta$-hydroxy-propionic acid is rapid, so that after a short time the solution is free from $\beta$-propiolactone. The completion of this hydrolysis can easily be recognised by the attainment of a constant $\mathrm{pH}$. Factors that decrease the esterase activity and rate of hydrolysis of $\beta$-propiolactone and thereby increase the possibility of protein modification are: heat, adsorption and strong complexing agents like EDTA. This is why EDTA plasmas lose their clotting activity on treatment with $0.3 \% \beta$-propiolactone.

Bei der Inaktivierung von Vaccinen (1) und der Herstellung von hepatitissicherem Plasma (2) und Serum (3) spielt $\beta$-Propiolacton eine bedeutende Rolle. Es interessierte die Frage, wie schnell und wie vollständig überschüssiges $\beta$-Propiolacton in Seren hydrolysiert, von welchen Faktoren diese Hydrolyse-Geschwindigkeit abhängt und unter welchen Bedingungen Proteinmodifizierungen auftreten.

Hydrolyse von $\beta$-Propiolacton in Serum und in physiologischer $\mathrm{NaCl}$-Lösung

$\mathrm{Da}$ die Hydrolyse von $\beta$-Propiolacton gemäß folgender Gleichung verläuft, stellt die Titration der bei dieser Reaktion auftretenden $\mathrm{H}^{+}$-Ionen ein gutes $\mathrm{Ma} \beta$ für den Ablauf der Hydrolyse dar.<smiles>O=C1CCO1</smiles><smiles>O=C([18O])CCO</smiles>

Bei unseren Versuchen arbeiteten wir bei konstantem $\mathrm{pH}$-Wert unter folgenden Bedingungen:

Einem 100 Spender-Serumpool wurden $0,3 \mathrm{~m} l \beta$-Propiolacton pro $100 \mathrm{~m} l$ Serum bei einem $\mathrm{pH}$-Wert von 8,0 zugefügt und die Hydrolyse bei $\mathrm{pH} 8,0$ und $37^{\circ}$ durchgeführt. Der Proteingehalt betrug $7,2 \mathrm{~g} / 100 \mathrm{ml}$. Es stellte sich heraus, daß die Hydrolyse von $\beta$-Propiolacton in diesem Serumpool im Gegensatz zu Einzelspender-Seren und -Plasmen grundsätzlich schneller verläuft als in 0,9 proz. NaCl-Lösung (Abb. 1).

Den Grund hierfür sehen wir im. Vorliegen von SerumEsterasen, die die Spaltung des inneren Esters $\beta$-Pro-

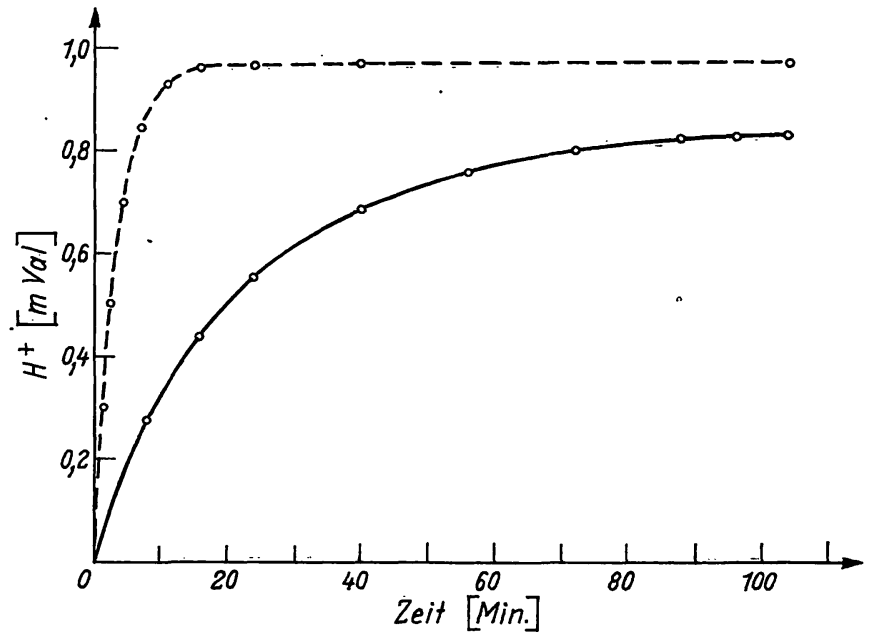

Abb. 1

Unterschiedliche Hydrolysegeschwindigkeit von $\beta=$ Propiolacton. in Serum und $\mathrm{NaCl}$-Lösung

$$
\text { 0,9 proz. NaCl-Lösung _..... Serum }
$$

piolacton beschleunigen (4). Die Halbwertszeit von $\beta$-Propiolacton in einem Serumpool beträgt unter den eingangs angegebenen Bedingungen etwa $3 \mathrm{Min}$; die Umwandlung des überschüssigen $\beta$-Propiolacton in die gut verträgliche $\beta$-Hydroxy-Propionsäure (5) ist nach etwa 1 Std. vollständig abgeschlossen. Ein hervorragendes Kriterium für den Abschluß dieser Hydrolyse ist der konstante $\mathrm{pH}$-Wert in einem solchen $\beta$-Propiolacton behandelten Serum, kenntlich daran, daß keine Natronlauge zur Neutralisation der entstehenden Säure verbraucht wird (s. Abb. 1). 
Wir haben an anderer Stelle darauf hingewiesen, daß ein Zusammenhang besteht zwischen Hydrolysegeschwindigkeit bzw. Verweilzeit von $\beta$-Propiolacton in Seren und Plasmen und dem Modifizierungsgrad der Proteine (6). Die Untersuchungen wurden damals an Einzelspender-Seren und -Plasmen durchgeführt. Es soll im folgenden der Einfluß von Erhitzen, Adsorption und Zusatz von Blutstabilisatoren auf die Hydrolysegeschwindigkeit von $\beta$-Propiolacton und damit auf die Proteinmodifizierungen in Serumpools gezeigt werden.

\section{Hydrolyse von $\beta$-Propiolacton in vorbebandelten Serumpools}

Einem 100 Spender-Serum wurden jeweils entweder $60 \mathrm{mVal} / \mathrm{l}$ Citrat oder $160 \mathrm{mg} / 100 \mathrm{~m} /$ EDTA zugesetzt. Ein anderer Teil des Serums wurde entweder durch Adsorption mit Aerosil oder Erhitzen auf $56^{\circ}$ vorbehandelt. Diese Seren wurden zusammen mit einer nicht vorbehandelten Probe unter den eingangs genannten Bedingungen mit $\beta$-Propiolacton zur Reaktion gebracht und die Halbwertszeiten bestimmt. Die Ergebnisse sind in Tabelle 1 zusammengefaßt.

Tab. 1

Halbwertszeiten von $\beta$-Propiolacton in vorbehandelten Seren

\begin{tabular}{clc}
\hline Nr. & \multicolumn{1}{c}{ Serumbehandlung } & $\begin{array}{c}\text { Halbwertszeit } \\
\text { (T/2) von } \\
\beta \text {-Propiolacton } \\
\text { (Min.) }\end{array}$ \\
\hline 1 & frisches Serum (100 Spender) unbehandelt & 2,4 \\
2 & Serum + Citrat (60 mVal $/ l)$ & 3,0 \\
3 & Serum + Erhitzen (30 Min. 56 $)$ & 6,0 \\
4 & Serum + Adsorption (4 Stdn 2\% & 30,0 \\
& Aerosil, 45 $)$ & 32,0 \\
6 & Serum + EDTA (160 mg/100 ml) & 2,4 \\
\hline
\end{tabular}

Die zuvor erwähnten Serumesterasen werden also offenbar durch die Vorbehandlungen mehr oder weniger direkt oder indirekt inaktiviert: direkt durch Erhitzen auf $56^{\circ}$ und durch Adsorption an Aerosil, indirekt durch CaBindung. Diese ist im Falle von Citrat nur schwach, bei EDTA jedoch außerordentlich stark ausgeprägt. Diese Blockierung durch EDTA kann wieder rückgängig gemacht werden durch Zusatz von $\mathrm{CaCl}_{2}$. Die $\mathrm{Halb}$ wertszeit in einem solchen recalcifizierten EDTA-Serum ist dann wieder normal, wie ebenfalls aus Tabelle 1 hervorgeht.

Die immunelektrophoretische Untersuchung der Seren der Tabelle 1 ergeben einen eindeutigen Zusammenhang zwischen Halbwertszeit (T/2) und Modifizierungsgrad. Je größer $T / 2$ um so größer ist die prozentuale Erhöhung der elektrophoretischen Wanderungsgeschwindigkeit infolge Erhöhung der negativen Ladung der Proteine durch Reaktion mit $\beta$-Propiolacton (Tab. 2).

Besonders wichtig ist der Befund, da $\beta$ die Behandlung von EDTA-Serum mit $\beta$-Propiolacton zu extrem starken Proteinmodifizierungen führt: Dies bedeutet offenbar, daß man EDTA-Plasmen im Gegensatz zu CitratPlasmen nicht nach der Standard-Methode mit $0,3 \%$ $\beta$-Propiolacton sterilisieren kann.
Tab. 2

Zusammenhang zwischen Halbwertszeit von $\beta$-Propiolacton und Proteinmodifizierung (Erhöhung der elektrophoretischen Wanderungsteinmodifizierung (Erhöhung der elektroph

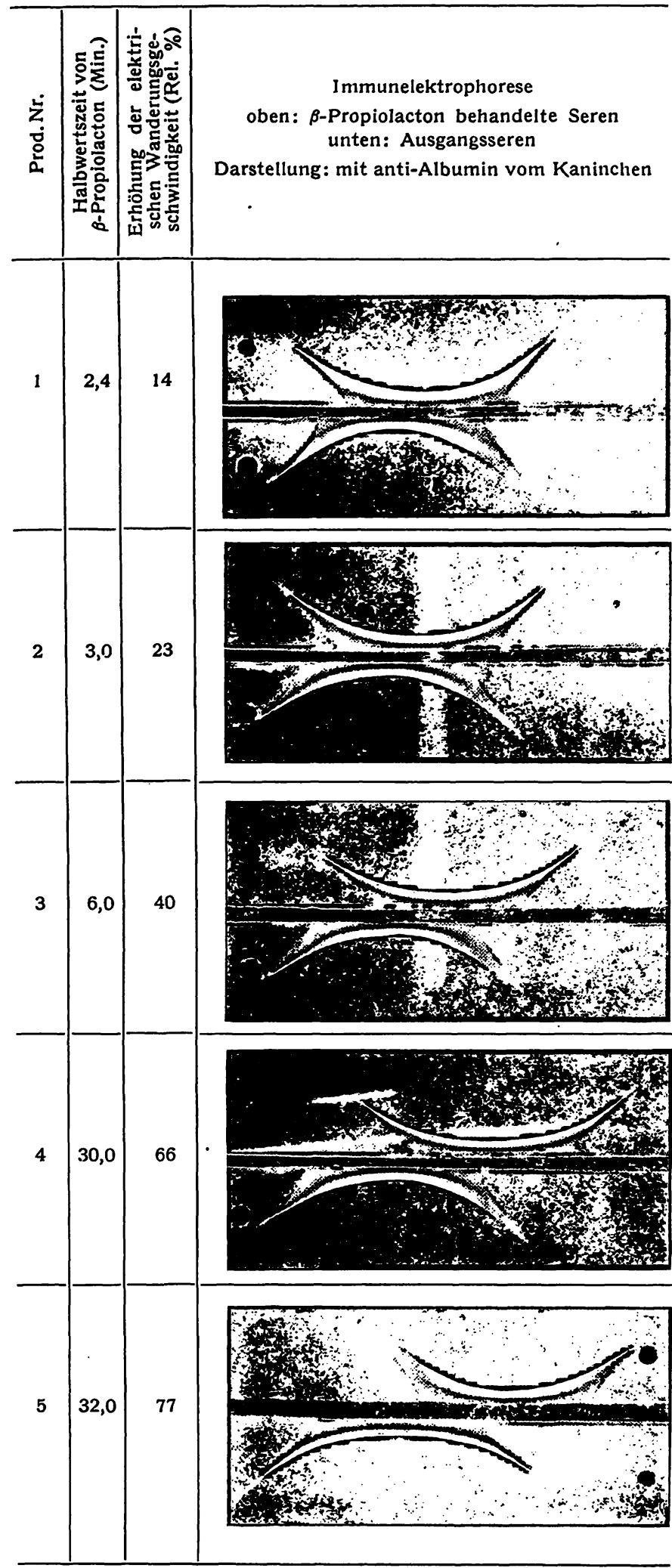

\section{Behandlung von EDT A-Plasma mit $\beta$-Propiolacton}

Behandelt man EDTA-Plasma (10 Spender-Pool) unter Standard-Bedingungen mit $\beta$-Propiolacton, so zeigt die Immunelektrophorese die erwartete starke Modifizierung. Dies gilt insbesondere für das Fibrinogen (s. Abb. 2). 


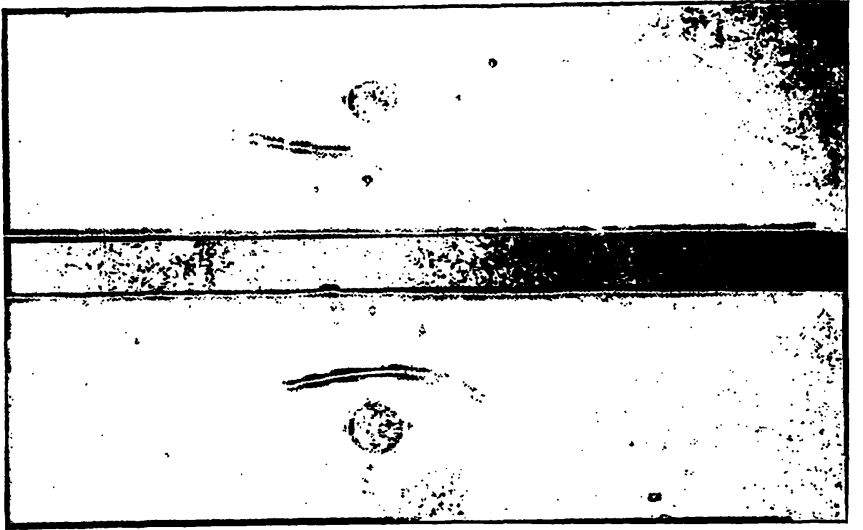

Abb. 2

Immunelektrophoretische Analyse von modifiziertem Fibrinogen Immunelektrophoretische $\beta$-Propiolacton behandeltes EDTA-Plasma (unten). Darstellung_mit anti-Fibrinogen vom Kaninchen

Das so veränderte Fibrinogen hat seine physiologische Aktivität verloren: Auf Zusatz von Thrombin tritt keine Gerinnung ein, während ein analog behandeltes Citrat-Plasma keinen Verlust seiner Gerinnungsaktivität zeigt ('Tab. 3).

Tab. 3

Gerinnungsaktivität von $\beta$-Propiolacton behandelten Citrat- und EDTA-Plasmen

\begin{tabular}{lcc}
\hline Plasma & $\begin{array}{c}\text { gerinnungsfähiges } \\
\text { vor } \beta \text {-Propiolacton- } \\
\text { Behandlung }\end{array}$ & $\begin{array}{c}\text { Finogen }(\mathrm{mg} / 100 \mathrm{ml}) \\
\text { nach } \beta \text {-Propiolacton- } \\
\text { Behandlung }\end{array}$ \\
\hline $\begin{array}{l}\text { Citrat-Plasma } \\
\text { (10 Spender) }\end{array}$ & 302 & 308 \\
$\begin{array}{l}\text { EDTA-Plasma } \\
\text { (10 Spender) }\end{array}$ & 383 & 0 \\
\hline
\end{tabular}

Unsere Befunde werden durch Doleschel und AuersWALD (7) bestätigt, die bei der Behandlung einer Lösung von isoliertem Fibrinogen mit $0,3 \% \beta$-Propiolacton einen weitgehenden Verlust der Gerinnungsaktivität feststellen:

Die Konzentration von $0,3 \% \beta$-Propiolacton ist eben bei Abwesenheit von $\beta$-Propiolacton-spaltenden Esterasen zu hoch, um Proteinmodifizierungen zu vermeiden. Weitere Versuche sollen zeigen, wie die $\beta$-PropiolactonKonzentration der Esterase-Aktivität anzupassen ist, damit keine Proteinmodifizierung eintritt und dennoch Sterilität und in Kombination mit UV-Bestrahlung Hepatitissicherheit Bewirkt wird.

\section{Beschreibung der Versuche}

\section{Hydrolyse von $\beta$-Propiolacton}

Die Bestimmung wirde mit einer Kombination von Titrigraph, Titrator und Autobürette der Fa. Radiometer, Kopenhagen, durchgeführt.

$20 \mathrm{~m} l$ Probe werden vorgelegt und bei $37^{\circ}$ auf $\mathrm{pH} 8,0$ eingestellt. Dann werden $0,3 \mathrm{~m} l \beta$-Propiolacton pro $100 \mathrm{~m} l$ Lösung zugefügt und bei $37^{\circ}$ mit $1 \mathrm{~N} \mathrm{NaOH}$ titriert.

\section{Behandlung von Plasma mit $\beta$-Propiolacton}

Citrat oder EDTA-Plasma wird auf $\mathrm{pH} 8,0$ eingestellt und bei $5^{\circ}$ $0,3 \mathrm{~m} l \beta$-Propiolacton pro $100 \mathrm{~m} l$ Lösung zugefügt. Man läßt $1 \mathrm{Std}$. bei $5^{\circ}$ stehen, stellt den $\mathrm{pH}$-Wert auf 8,0 nach und beendet die Hydrolyse bei $37^{\circ}$ und $\mathrm{pH} 8,0$.

\section{Immunelektrophorese}

Es wurde wie üblich nach Grabar in der Modifikation von SCHEIDEGGER unter Verwendung von anti-Fibrinogen und anti-Albumin (Behringwerke, Marburg) gearbeitet.

Plasmaverdünnung: $1: 8$

Serumverdünnung: $1: 2$

\section{Fibrinogen-Bestimmung}

Reagenzien: $35 \mathrm{ml} 0,9$ proz. NaCl-Lösung $1 \mathrm{~m} / 25 \mathrm{~mm} \mathrm{CaCl}$-Lösung $0,5 \mathrm{ml} 0,1$ proz. wäßr. Thrombinlösung.

$1,0 \mathrm{~m} l$ Plasma wird in ein $50 \mathrm{~m} /$-Zentrifugenglas einpipettiert, mit den angegebenen Mengen Reagenzien zur Durchmischung leicht bewegt und $1 \mathrm{Std}$. im Wasserbad bei $37^{\circ}$ inkubiert. Anschließend wird $20 \mathrm{Min}$. bei $5000 \mathrm{U}$./Min. zentrifugiert. Das am Boden des Glases zusammengeballte Gerinnsel wird mit einem PVC-Stäbchen sauber herausgefischt und auf einem Filtrierpapier getrocknet. Das ausgedrückte Gerinnsel wird mit Wasser abgespült und nochmals auf Filtrierpapier abgepreßt.

$\mathrm{Zu}$ dem in ein Wassermannröhrchen abgestreiften Gerinnsel werden $2,5 \mathrm{~m} l 10$ proz. $\mathrm{NaOH}$ hinzupipettiert. Zur vollständigen Auflösung wird $30 \mathrm{Min}$. bei $37^{\circ}$ inkubiert. Nach Zusatz von $2,5 \mathrm{ml}$ Biuret-Lösung wird der Proteingehalt gegen eine Mischung aus 10 proz. $\mathrm{NaOH}$ und Biuretlösung im Verhältnis 1:1 bestimmt.

Frl. U. REXrodT und Frl. U. FrAHM danke ich für ihre technische Assistenz.

\section{Literatur}

1. Fayet, M. T., H. G. Petermann, J. Fontaine, J. Terre und M. Roumiantzeff, Ann. Inst. Pasteur Paris 112, 65 (1967). 2. HartmanN, F. W. und G. A. LoGrippo, in „Hepatitis Frontiers", Seite 407, Henry Ford Hospital International Symposium Churchill Ltd., London (1957). - 3. Stephan, W. und G. MAY, diese Z. 6, 191 (1968). - 4. Stephan, W. und L. RókA, diese Z.
6, 186 (1968). - 5. Kelly, A. R., F. W. Hartmann und C. E. RUPE, in „Hepatitis Frontiers”, Seite 307, Henry Ford Hospital International Symposium Churchill Ltd., London (1957). 6. Stephan, W., diese Z. 6, 481 (1968). - 7. Doleschel, W. und W. Auerswald, Pharmacology 2, 1 (1969).

Dr. W. Stephan BIOTEST-Serum-Institut GmbH 6 Frankfurt (Main)

Flughafenstr. 4 


\section{Are you in doubt?}
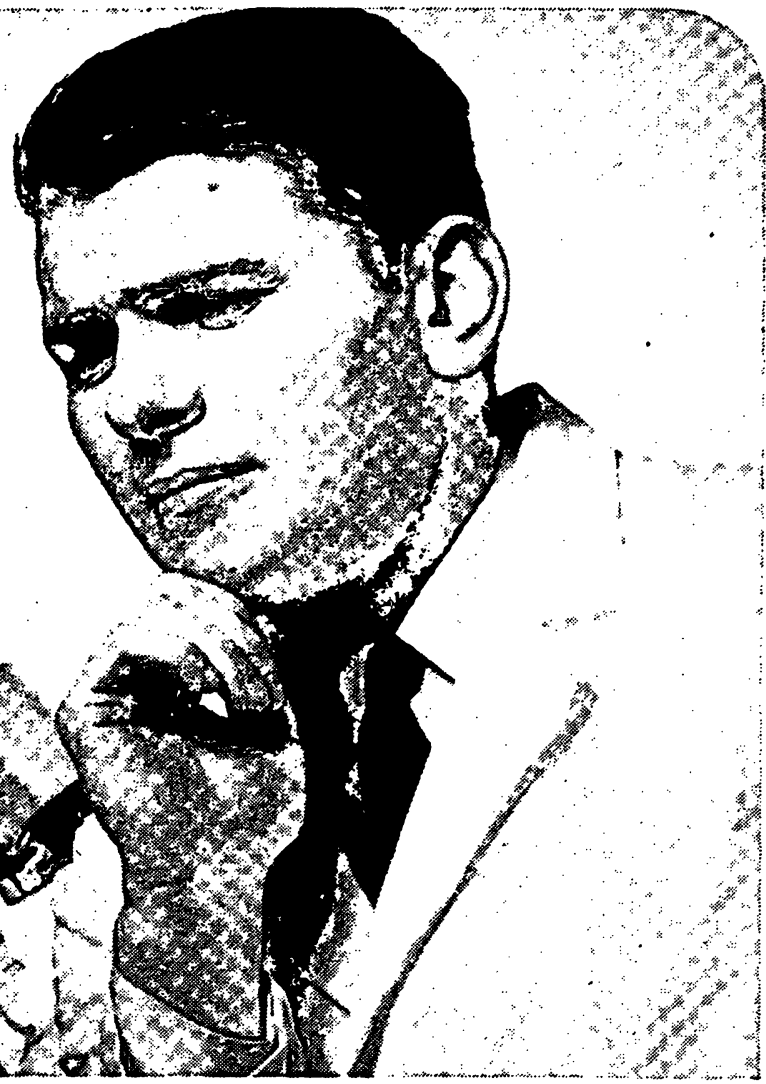

\section{Seronorm gives you confidence.}

- Seronorm is a stable, freeze-dried standard serum.

- Seronorm controls the method, the equipment, the reagents and the analysts:

- Analytical data are given for about 25 serum substances in Seronorm, determined according to approx. 50 generally accepted analytical methods, by the following laboratories:

A. Institute of Clinical Biochemistry, Oslo University Hospital, Oslo.

B. The Central Laboratory, Copenhagen University Hospital, Copenhagen.

C. The Central Laboratory, Karolinska Sjukhuset, Stockholm.

D. The Central Laboratory, The Växjö Regional Hospital, (Centrallasarettet), Växjö, (Sweden).

The results of the analyses are given separately for each method.

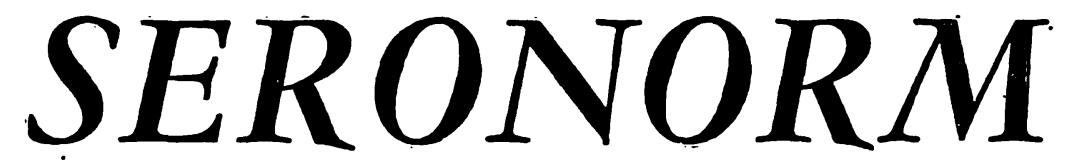

NYEGAARD\& CO.AS.OSLO.NORWAY 


\section{Sephadex in}

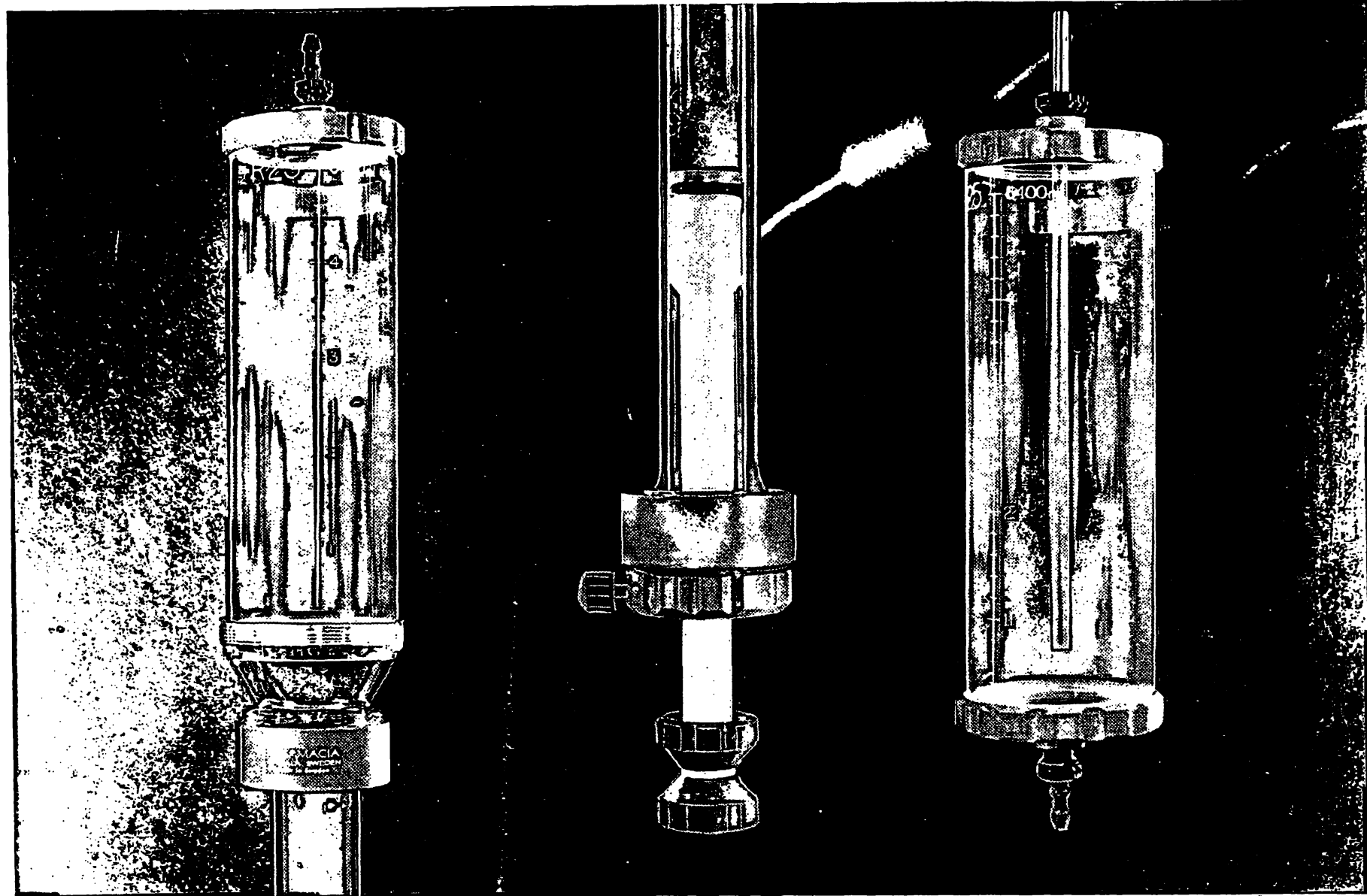

\section{ARC der Säulenchromatographie}

A Packen mit neuen Gel- und Eluant-Reservoiren

Die neuen Reservoire haben wir speziell für die Gelfiltration entwickelt. Auf das Chromatographierohr aufgeschraubt machen sie das Packen zu einem automatischen Vorgang. Das Ergebnis: Gleichmäßig gepackte Gelfüllungen.

Reservoire können Sie für jeden Typ unserer Chromatographierohre bestellen.

B Probenauftrag mit Durchflußadaptern

Die Adapter werden für den automatischen und reproduzierbaren Probenauftrag benutzt. Das Er- gebnis: Gleichmäßige Verteilung der Probe über die Oberfläche der Füllung.

C Elution mit neuen Gel- und Eluant-Reservoiren

Die neuen Reservoire können in eine Mariottesche Flasche umgewandelt werden. Sie ermöglichen dann automatisches Äquilibrieren und Elution bei konstantem Arbeitsdruck. Mit Durchflußadaptern können Sie aufsteigend, absteigend oder zyklisch chromatographieren.

Wir informieren Sie gern über unsere Lieferprogramme an Chromatographierohren und Zubehör. Bitte schreiben Sie uns. 